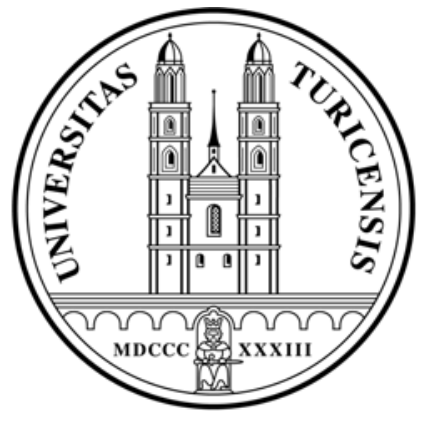

Institute for Empirical Research in Economics

University of Zurich

Working Paper Series

ISSN 1424-0459

Working Paper No. 383

Preference Reversals and Probabilistic Choice

Pavlo R. Blavatskyy

August 2008 


\title{
Preference Reversals and Probabilistic Choice
}

\author{
Pavlo R. Blavatskyy \\ University of Zurich (IEW) \\ Winterthurerstrasse 30 \\ CH-8006 Zurich \\ Switzerland \\ Phone: $+41(0) 446343586$ \\ Fax: $\quad+41(0) 446344978$ \\ e-mail: pavlo.blavatskyy@,iew.uzh.ch
}

August 2008

\begin{abstract}
:
Preference reversals occur when different (but formally equivalent) elicitation methods reveal conflicting preferences over two alternatives. This paper shows that when people have fuzzy preferences i.e. when they choose in a probabilistic manner, their observed decisions can generate systematic preference reversals. A simple model of probabilistic choice and valuation can account for a higher incidence of standard (nonstandard) preference reversals for certainty (probability) equivalents and it can also rationalize the existence of strong reversals. An important methodological contribution of the paper is a new definition of a probabilistic certainty/probability equivalent of a risky lottery.
\end{abstract}

Keywords: preference reversal, probabilistic choice, certainty equivalent, probability equivalent, valuation

JEL classification codes: D01, D80, D81, C91 


\section{Preference Reversals and Probabilistic Choice}

Any successful descriptive theory of choice and valuation will need to allow in some way for the imprecision surrounding people's decisions.

David Butler and Graham Loomes (2007), AER 97(1), p.277

\section{The Preference Reversal Phenomenon}

The preference reversal phenomenon was first documented by Sarah Lichtenstein and Paul Slovic (1971). It was subsequently replicated in dozens of studies (see Seidl (2002) for a recent review). A typical illustration of the preference reversal phenomenon involves two risky lotteries of a similar expected value. The first lottery offers a relatively large sum of money with a small probability and zero otherwise. This lottery is usually called the $\$$-bet. The second lottery yields a modest sum of money with a relatively high probability and zero otherwise. This lottery is called the P-bet.

A standard preference reversal is observed when a decision maker chooses the P-bet over the \$-bet in a direct binary choice and at the same time he or she states a higher certainty equivalent for the $\$$-bet in the valuation task. A nonstandard preference reversal occurs when a decision maker chooses the \$-bet over the P-bet while stating a higher certainty equivalent for the P-bet. The preference reversal phenomenon is an observation that standard preference reversals occur more frequently than nonstandard preference reversals. In a broader sense, the preference reversal phenomenon occurs when a decision maker reveals different preferences (in a predictable manner) in two elicitation procedures that are formally equivalent. The phenomenon is often interpreted as the failure of procedure invariance (e.g. Tversky et al., 1990).

The preference reversal phenomenon can also occur when an individual chooses the P-bet over the \$-bet in a direct binary choice even though the certainty equivalent of the $\$$-bet is strictly greater than the highest possible outcome of the P-bet. 
Such preference reversals are called strong reversals (Fishburn, 1988, p.46). Several non-expected utility theories such as regret theory (e.g. Loomes and Sugden, 1987) can explain the preference reversal phenomenon as a consequence of non-transitive preferences but they cannot rationalize strong reversals.

Butler and Loomes (2007) reported a new form of the preference reversal phenomenon using probability equivalents instead of certainty equivalents. In this case, a standard preference reversal occurs when a decision maker chooses the P-bet over the \$-bet while stating a higher probability equivalent for the \$-bet. A nonstandard preference reversal is observed when the \$-bet is chosen over the P-bet but the probability equivalent of the P-bet is higher. Butler and Loomes (2007) find that such nonstandard preference reversals occur more frequently than standard preference reversals when probability equivalents are used in the valuation task.

The preference reversal phenomenon provides direct evidence that people reveal their preferences through their observed decisions with some degree of noise or imprecision (e.g. Butler and Loomes, 2007). If the observed decisions were always only a result of conscious utility maximization, both standard and nonstandard preference reversals would never occur (unless a decision maker is exactly indifferent between the \$-bet and the P-bet). When decisions reflect deliberate preference maximization with some error, or underlying preferences are imprecise, both types of preference reversals are possible. This paper shows that a model of probabilistic choice and valuation can account for a higher incidence of standard preference reversals with certainty equivalents simultaneously with a higher incidence of nonstandard preference reversals with probability equivalents. The model can also rationalize the existence of strong reversals.

To illustrate the main idea of the paper let us consider the following example. 
Example 1 The P-bet yields $\$ 3.75$ with a probability 0.8 (nothing otherwise) and the \$-bet yields \$10 with a probability 0.3 (\$0 otherwise). Notice that these bets have the same expected value (\$3). Suppose that an individual chooses with equal probabilities between these two bets in a direct binary choice. According to the model presented in this paper, the certainty equivalents of the \$-bet and the P-bet are random variables with the following intuitive properties:

1) the certainty equivalent of the $\$$-bet is distributed between $\$ 0$ and $\$ 10$;

2) the certainty equivalent of the P-bet is distributed between $\$ 0$ and $\$ 3.75$;

3) a median certainty equivalent of the $\$$-bet is equal to that of the P-bet (\$3).

Note that these properties imply that the distribution of the certainty equivalent of the \$-bet (the P-bet) is positively (negatively) skewed as it is illustrated on Figure 1. If we take two draws from such distributions, we can observe many instances when the realized certainty equivalent of the $\$$-bet is greater than the realized certainty equivalent of the P-bet and only few instances of the reversed ranking. Thus, an individual ends up stating a systematically higher certainty equivalent for the \$-bet (“overpricing error").

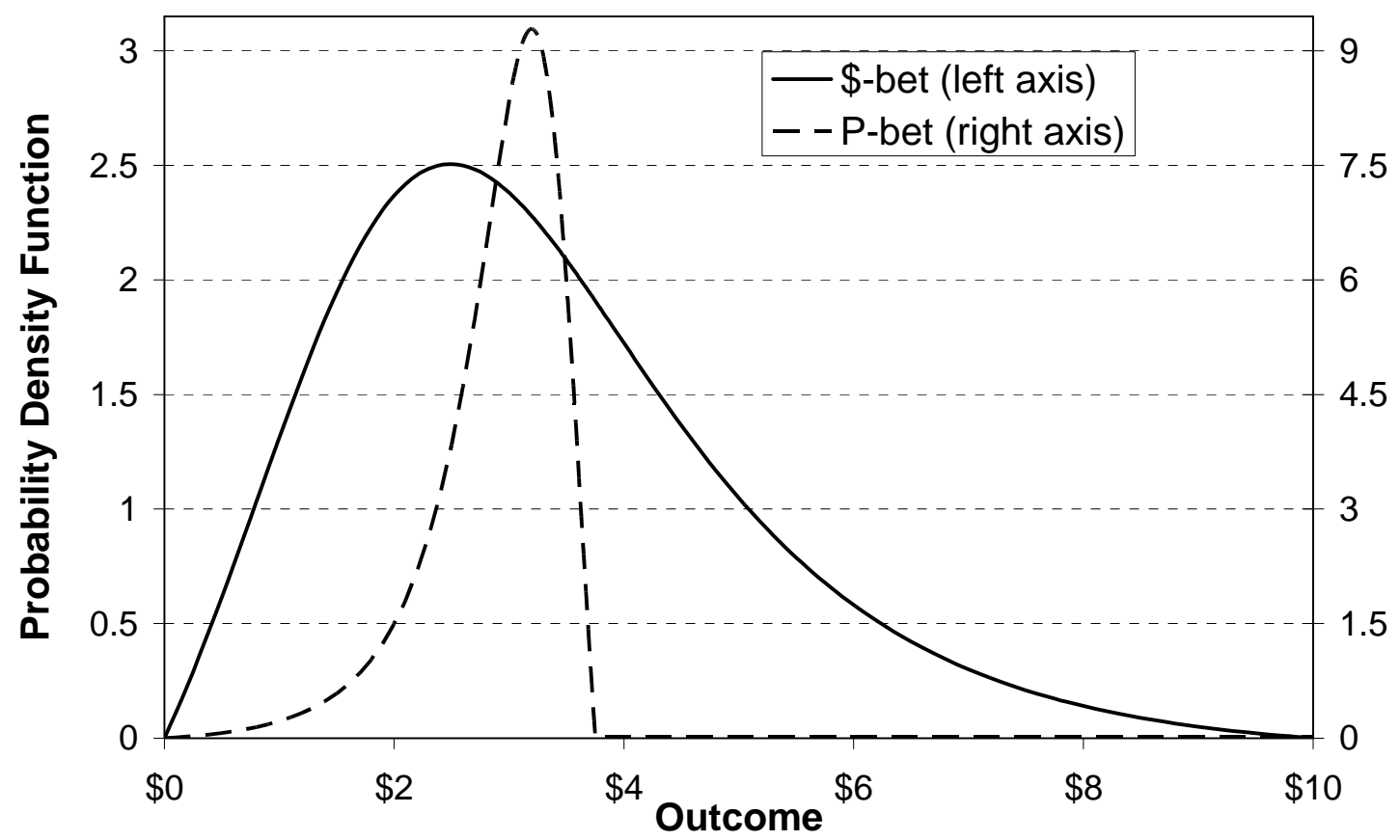

Figure 1 Probability density function of the certainty equivalent of the \$-bet and the P-bet 
The idea that preference reversals may be driven by random preferences or random errors has been around for quite a while. MacCrimmon and Smith (1986) were among the first who suggested that imprecise certainty and probability equivalents can account for the preference reversal phenomenon. Starting with a general premise "it appears that people are unable to determine precise equivalences for lotteries" (MacCrimmon and Smith, 1986, p.13) they moved on to demonstrate that "the imprecise equivalence theory explains traditional preference reversal $\ldots$ in terms of the spread of the certainty equivalence for the \$-bet” (MacCrimmon and Smith, 1986, p.15).

The ideas of MacCrimmon and Smith (1986) were recently reiterated in Butler and Loomes (2007). However, neither MacCrimmon and Smith (1986) nor Butler and Loomes (2007) developed a tractable mathematical model that formalizes the concept of imprecise certainty and probability equivalents. The present paper contributes to this literature by providing a formal definition of probabilistic certainty/probability equivalents within the framework of a general model of probabilistic choice.

Looking at the phenomenon from a different perspective, Schmidt and Hey (2004) found that preference reversals occur less frequently if people reveal consistent ordering of two alternatives in repeated pricing tasks. Hence, Schmidt and Hey (2004) suggest that the preference reversal phenomenon can be driven by random errors in the elicitation of certainty equivalents. The model presented in this paper is consistent with such interpretation (cf. Example 1 above).

The remainder of the paper is organized as follows. Section 2 presents a simple model of probabilistic choice and defines a probabilistic certainty/probability equivalent of a risky lottery. Section 3 shows that this model can account for classical preference reversals (subsection 3.1), strong preference reversals (subsection 3.2) and preference reversals with probability equivalents (subsection 3.3). Section 4 concludes. 


\section{A Model of Probabilistic Choice and Valuation}

Experimental studies of repeated decision making under risk demonstrate that individual choices are often contradictory. For instance, Hey and Orme (1994) find that around $25 \%$ of observed choice decisions are reversed when subjects are presented with the same decision problem for the second time and they have a possibility to declare indifference. Ballinger and Wilcox (1997) report the median switching rate of $20.8 \%$. While people generally choose in a probabilistic manner between risky lotteries, they seldom violate transparent dominance (e.g. Loomes and Sugden, 1998; Hey, 2001).

Hence, we begin by considering models of probabilistic choice that prohibit violations of transparent dominance. In particular, we do not consider a strong utility model, which includes a Fechner model of random errors (e.g. Hey and Orme, 1994) and Luce choice model as special cases, because it allows for violations of dominance. There are essentially four models of probabilistic decision making proposed in the literature that respect stochastic dominance:

1) incremental expected utility advantage model (Fishburn, 1978);

2) random preference model (Loomes and Sugden, 1995);

3) random expected utility model (e.g. Gul and Pesendorfer, 2006);

4) model of probabilistic choice proposed by Blavatskyy (2008).

A straightforward application of random preference or random utility model to the preference reversal phenomenon is problematic because we need to consider utilities of (infinitely) many outcomes when eliciting certainty equivalents of the \$-bet and the P-bet. We can restrict our attention to a specific parsimonious parametric form of random preference/utility model. However, then it is not clear if our drawn conclusions generalize to a generic random preference/utility model. Due to these considerations, we do not explicitly employ random preference/utility framework in this paper. However, a 
model of probabilistic choice that we consider below is consistent with the idea that a decision maker does not have a unique preference relation and/or the observed decisions are described by a random utility model.

Being left with a choice between Fishburn (1978) and Blavatskyy (2008), it appears that the latter model is more descriptively adequate. The model of Fishburn (1978) is incompatible with the common ratio effect (Allais, 1953) because it relies on the stochastic analogue of the independence axiom. However, the model of Blavatskyy (2008) uses a weaker axiom and it can account for the common ratio effect.

Thus, in this paper we will consider a model of probabilistic choice where an individual chooses a lottery $A$ over another lottery $B$ with a probability

$$
P(A, B)=\frac{\varphi(U(A)-U(A \wedge B))}{\varphi(U(A)-U(A \wedge B))+\varphi(U(B)-U(A \wedge B))},
$$

where $U($.$) is the von Neumann-Morgenstern expected utility function, \varphi: \mathbb{R}_{+} \rightarrow \mathbb{R}_{+}$is a non-decreasing function with $\varphi(0)=0$ and $A^{\wedge} B$ denotes the greatest lower bound on $A$ and $B$ in terms of weak stochastic dominance. Blavatskyy (2008) provides an axiomatic characterization of the choice rule (1).

Model (1) has many intuitive properties. An individual always chooses $A$ over $B$ if $A$ stochastically dominates $B$ (in this case $B=A^{\wedge} B$ ). Similarly, an individual never chooses $A$ over $B$ if $B$ stochastically dominates $A$ (in this case $A=A^{\wedge} B$ ). An individual chooses $A$ over $B$ with a probability greater than or equal to 0.5 if $U(A) \geq U(B)$.

Model (1) is consistent with the common ratio effect (Allais, 1953) and the violations of the betweenness axiom (e.g. Blavatskyy, 2006) if function $\varphi($.$) is non-$ homogeneous. Random expected utility model (Gul and Pesendorfer, 2006) is a special case of model (1) when lotteries $A$ and $B$ involve no more than three distinct outcomes (as in a binary choice between the $\$$-bet and the P-bet) and if function $\varphi($.$) is$ 
homogeneous. Finally, model (1) can also have an error interpretation: the observed decisions are not always a direct result of deliberate preference maximization but they can also reflect random errors or noise (e.g. Hey and Orme, 1994; Blavatskyy, 2007).

The preference reversal phenomenon involves two decision tasks - a binary choice between lotteries and the valuation task (i.e. the elicitation of either certainty or probability equivalents). Thus, beside model (1) of binary choice we also need to define a certainty/probability equivalent of a risky lottery.

\subsection{Certainty Equivalent of a Lottery}

Lottery valuation/pricing is closely related to a binary choice between a lottery and an amount for certain. When people have fuzzy preferences it seems natural to assume that the certainty equivalent of a risky lottery is a random variable. Such assumption is supported by experimental evidence on repeated pricing. Blavatskyy and Köhler (2007) found that only $16.7 \%$ of subjects consistently state the same price for identical lotteries when the pricing task is repeated for the second time.

Definition 1 The certainty equivalent of a lottery $A$ is a random variable with the cumulative distribution function

$$
C E_{A}(x)=P(x, A)=1-P(A, x) .
$$

According to Definition 1, the probability that the certainty equivalent of a risky lottery is less than or equal to an amount $x$ is simply the probability that the amount $x$ is chosen over the lottery in a direct binary choice. Thus, the more likely is an individual to choose an amount $x$ over the lottery, the more likely it is that the certainty equivalent of this lottery is less than $x$. Note that $C E_{A}(x)=0$ for any amount $x$ that is less than the lowest possible outcome of lottery A. In other words, the certainty equivalent of a lottery cannot be less than the lowest possible outcome of a lottery. Similarly, the certainty equivalent cannot be greater than the highest possible outcome of the lottery. 


\subsection{Probability Equivalent of a Lottery}

The probability equivalent of a lottery $A$ is elicited by comparing lottery $A$ to a benchmark two-outcome lottery $Q$ that yields a lower outcome with a probability 1- $q$ and a higher outcome with a probability $q$. The lower outcome of a benchmark lottery is usually the same as the lowest possible outcome of lottery A. The higher outcome of a benchmark lottery is usually greater than the highest possible outcome of lottery $A$. When people have deterministic preferences, the probability equivalent of $A$ is traditionally defined as a probability $q$ such that an individual finds the benchmark lottery $Q$ exactly as good as the lottery $A$. When people have fuzzy preferences it seems natural to assume that the probability equivalent of a lottery is a random variable.

Definition 2 The probability equivalent of a lottery $A$ is a random variable with the cumulative distribution function

$$
P E_{A}(q)=P(Q, A)=1-P(A, Q) .
$$

Similar as for a certainty equivalent, we define the cumulative distribution function of a probability equivalent in the following way. The likelihood that the probability equivalent of a lottery $A$ is less than or equal to $q$ is simply the probability that the benchmark lottery $Q$ is chosen over $A$ in a direct binary choice. Thus, the more likely is an individual to choose the benchmark lottery $Q$ over $A$, the more likely it is that the probability equivalent of $A$ is less than $q$.

As any probability number, a probability equivalent must be bounded between zero and one. If the lower outcome of a benchmark lottery is the same as the lowest possible outcome of lottery $A$, formula (3) implies that $P E_{A}(0)=0$. If the higher outcome of a benchmark lottery is greater than the highest possible outcome of lottery A, formula (3) implies that $P E_{A}(1)=1$. Thus, according to Definition 2, a probability equivalent always lies between zero and one. 


\section{Preference Reversals Resulting from Probabilistic Choice}

\subsection{Classical Preference Reversal Phenomenon}

Let lottery $A$ denote the P-bet and lottery $B$ denote the \$-bet. In a direct binary choice, an individual chooses the $\$$-bet over the P-bet with a probability $p_{1}=P(B, A)$. The same individual states a higher certainty equivalent for the $\$$-bet than for the P-bet with a probability

$$
p_{2}=\int C E_{A}(x) \mathrm{d} C E_{B}(x)=\int P(x, A) \mathrm{d} P(x, B) .
$$

A standard preference reversal is observed with a probability $\left(1-p_{1}\right) \cdot p_{2}$. A nonstandard preference reversal is observed with a probability $p_{1} \cdot\left(1-p_{2}\right)$. Therefore, standard preference reversals occur more often than nonstandard preference reversals if and only if $p_{2}>p_{1}$, which is equivalent to

$$
\int P(x, A) \mathrm{d} P(x, B)>P(B, A) .
$$

Let us now introduce the following notation. A relatively safe lottery $A$ (the Pbet) yields an outcome $y>0$ with a probability $s \in(0,1)$ and zero with a probability $1-s$ (with $s$ being for "safe"). A relatively risky lottery $B$ (the $\$$-bet) yields an outcome $z>y$ with a probability $r \in(0, s)$ and zero with a probability $1-r$ (with $r$ being for "risky").

An individual, who does not violate transparent dominance, never chooses an outcome $x \leq 0$ over lottery $A$ in a direct binary choice i.e. $P(x, A)=0$ for all $x \leq 0$. Similarly, such an individual always chooses an outcome $x \geq y$ over lottery $A$ in a direct binary choice i.e. $P(x, A)=1$ for all $x \geq y$. Using these two results it is possible to rewrite condition (5) as follows

$$
\int_{0}^{y} P(x, A) \mathrm{d} P(x, B)>P(B, A)-P(B, y) .
$$

Finally, using formula (1) we can spell out condition (6) into 


$$
\begin{gathered}
\int_{0}^{y} \frac{\varphi((1-s) u(x))}{\varphi((1-s) u(x))+\varphi(s[u(y)-u(x)])} \mathrm{d} \frac{\varphi((1-r) u(x))}{\varphi((1-r) u(x))+\varphi(r[1-u(x)])}> \\
>\frac{\varphi(r[1-u(y)])}{\varphi(r[1-u(y)])+\varphi((s-r) u(y))}-\frac{\varphi(r[1-u(y)])}{\varphi(r[1-u(y)])+\varphi((1-r) u(y))},
\end{gathered}
$$

where $u(x)$ denotes a Bernoulli utility function of outcome $x$, with a normalization $u(0)=0$ and $u(z)=1$. Condition (7) can be easily satisfied for plausible parameter values as it is demonstrated by the following example.

Example 2 If function $\varphi($.$) is linear and the \$-bet and the P-bet yield the same$ expected utility i.e. $u(y)=r / s$ then condition (7) becomes

$$
\ln \frac{1+s-2 r}{1-s}>2 \frac{s-r}{1-r}
$$

which is satisfied for all $r<s$.

There is a simple intuition behind Example 2 (e.g. MacCrimmon and Smith, 1986; Butler and Loomes, 2007). If the \$-bet and the P-bet have the same expected utility then an individual chooses any of them with a probability 0.5 in a direct binary choice. Moreover, in this case, median certainty equivalents of the \$-bet and the P-bet are exactly equal (in particular, they are implicitly defined by $u(x)=r$ ).

However, the distribution of the certainty equivalent of the \$-bet is positively skewed since its support is $[0, z]$ due to restrictions imposed by transparent dominance. At the same time, the distribution of the certainty equivalent of the P-bet is negatively skewed because its support is $[0, y]$. Hence, it is entirely possible that the certainty equivalent of the \$-bet happens to be greater than the certainty equivalent of the P-bet more often than vice versa. In fact, it is even possible to draw a stronger conclusion, as we illustrate in the next subsection. 


\subsection{Strong Reversals}

The model of probabilistic choice presented in Section 2 can also rationalize the existence of strong preference reversals when an individual chooses the P-bet over the $\$$-bet in a direct binary choice even though he or she states the certainty equivalent for the $\$$-bet which is greater than the highest possible outcome of the P-bet. The highest possible outcome of the P-bet is $y$. The probability that the certainty equivalent of the $\$$-bet is strictly greater than $y$ is $1-C E_{B}(y)=P(B, y)$. Therefore, the probability of observing a strong reversal is $\left(1-p_{1}\right) \cdot P(B, y)$. We already established that the probability of observing a nonstandard preference reversal is $p_{1} \cdot\left(1-p_{2}\right)$. Thus, strong reversals are more likely to occur than nonstandard preference reversals if $\left(1-p_{1}\right)$. $P(B, y)>p_{1} \cdot\left(1-p_{2}\right)$, which is equivalent to

$$
\int P(x, A) \mathrm{d} P(x, B)>1-P(A, B) \cdot P(B, y) / P(B, A) .
$$

If a binary choice probability function $P(.,$.$) always respects transparent$ dominance i.e. if $P(x, A)=0$ for all $x \leq 0$ and $P(x, A)=1$ for all $x \geq y$, then we can rewrite condition (9) as follows

$$
\int_{0}^{y} P(x, A) \mathrm{d} P(x, B)>1-\frac{P(B, y)}{P(B, A)} .
$$

Finally, given a specific model (1) of probabilistic choice, we can spell out condition (10) into

$$
\begin{gathered}
\int_{0}^{y} \frac{\varphi((1-s) u(x))}{\varphi((1-s) u(x))+\varphi(s[u(y)-u(x)])} d \frac{\varphi((1-r) u(x))}{\varphi((1-r) u(x))+\varphi(r[1-u(x)])}> \\
>\frac{\varphi((1-r) u(y))-\varphi((s-r) u(y))}{\varphi(r[1-u(y)])+\varphi((1-r) u(y))} .
\end{gathered}
$$

It turns out that condition (11) can be satisfied for typical parameterizations that are used in the empirical studies on the preference reversal phenomenon. For illustration, let us again consider Example 2. 
Example 2 (continued) If function $\varphi($.) is linear and the \$-bet and the P-bet yield the same expected utility then condition (11) becomes

$$
\ln \frac{1+s-2 r}{1-s}>2 \frac{s-r}{1-r} \frac{1+2 s-3 r}{1+s-2 r}
$$

The left hand side of inequality (12) becomes unboundedly large if probability $s$ gets close to one. However, the right hand side of inequality (12) cannot be larger than three if $r<s$. Hence, condition (12) is satisfied when probability $s$ is sufficiently close to one and probability $r$ is not too large (compared to $s$ ).

The set of all parameters $(s, r)$ that satisfy inequality (12) is shown as a shaded area on Figure 2. Figure 2 also plots parameters $(s, r)$ used in the experiment of Tversky et al. (1990), which were subsequently reemployed in several other studies (e.g. Cubitt et al., 2004; Blavatskyy and Köhler, 2007). These typical parameterizations largely fall in the area of admissible parameters, which are consistent with a high incidence of strong preference reversals (under extremely simplifying assumptions that function $\varphi($. is linear and the \$-bet and the P-bet yield the same expected utility). Thus, the model of probabilistic choice presented in Section 2 can account for strong preference reversals.

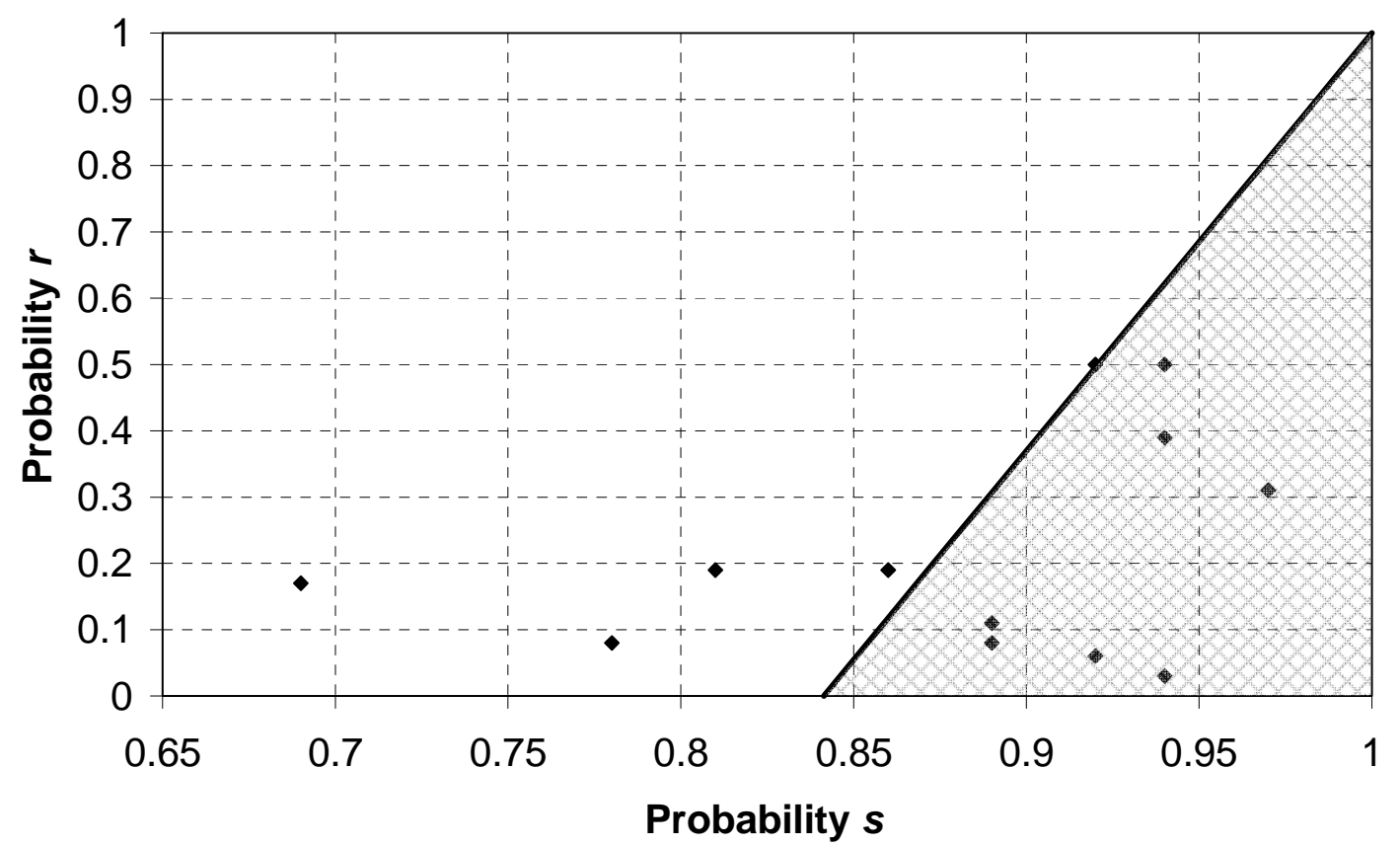

Figure 2 The set of parameters satisfying condition (12) (shaded area) and parameters used in Tversky et al. (1990) study (diamond markers) 


\subsection{Preference Reversals with Probability Equivalents}

The model of probabilistic choice presented in Section 2 can also account for a higher incidence of nonstandard preference reversals documented by Butler and Loomes (2007), when an individual chooses the \$-bet over the P-bet in a direct binary choice but at the same time he or she states a higher probability equivalent for the P-bet. In a direct binary choice, an individual chooses the $\$$-bet over the P-bet with a probability $p_{1}=$ $P(B, A)$. The likelihood that the probability equivalent of the P-bet is greater than the probability equivalent of the $\$$-bet is given by

$$
p_{3}=\int P E_{B}(q) \mathrm{d} P E_{A}(q) .
$$

Hence, nonstandard preference reversals occur with a probability $p_{1} \cdot p_{3}$. At the same time, standard preference reversals happen with a probability $\left(1-p_{1}\right) \cdot\left(1-p_{3}\right)$. Nonstandard preference reversals occur more often than the standard ones if and only if $p_{3}>1-p_{1}$, which is equivalent to

$$
\int P E_{B}(q) \mathrm{d} P E_{A}(q)>P(A, B) .
$$

If an individual does not violate transparent dominance, the probability equivalent of the $\$$-bet cannot be greater than $r$ i.e. $P E_{B}(q)=1$ for all $q \geq r$. Using this result we can rewrite inequality (14) as follows

$$
\int_{0}^{r} P E_{B}(q) \mathrm{d} P E_{A}(q)>P(A, B)-P(A, R),
$$

where $R$ denotes a benchmark lottery that yields a higher outcome with a probability $r$ (and zero with a probability $1-r$ ). Finally, if binary choice probabilities admit representation (1), we can spell out condition (15) into

$$
\begin{gathered}
\int_{0}^{r} \frac{\varphi(q[v-1])}{\varphi(q[v-1])+\varphi(r-q)} \mathrm{d} \frac{\varphi(q[v-u(y)])}{\varphi(q[v-u(y)])+\varphi((s-q) u(y))}> \\
>\frac{\varphi((s-r) u(y))}{\varphi((s-r) u(y))+\varphi(r[1-u(y)])}-\frac{\varphi((s-r) u(y))}{\varphi((s-r) u(y))+\varphi(r[v-u(y)])},
\end{gathered}
$$


where $v>1$ denotes the utility of a higher outcome in the benchmark lottery that is used for eliciting probability equivalents. Condition (16) can hold for plausible parameter values. To demonstrate this, let us return to Example 2.

Example 2 (continued) If function $\varphi($.$) is linear and the \$-bet and the P-bet$ yield the same expected utility then condition (16) becomes

$$
\ln \frac{s(v+1)-2 r}{s(v-1)}>2 \frac{s-r}{s v-r},
$$

which is satisfied for all $r<s$ and all $v>1$.

There is a simple intuition behind this result. If the expected utilities of the $\$-$ bet and the P-bet are the same, then an individual chooses with probabilities $50 \%-50 \%$ between them. Moreover, a median probability equivalent of the $\$$-bet is the same as that of the P-bet (in particular, it is equal to $r / v$ ). However, distributions of probability equivalents of the \$-bet and the P-bet are likely to be skewed in opposite directions. On the one hand, the distribution for the $\$$-bet is likely to be negatively skewed because it has support on $[0, r]$. On the other hand, the distribution for the P-bet is positively skewed because it has support on $[0, s]$. Thus, it is possible that an individual states a higher probability equivalent for the P-bet more often than he or she does for the \$-bet.

Notice that inequality (16) may hold simultaneously with inequality (7), as it is illustrated in Example 2. In other words, the model of probabilistic choice presented in Section 2 can account for a higher incidence of standard preference reversals for certainty equivalents simultaneously with a higher incidence of non-standard preference reversals for probability equivalents. In contrast, Cubitt et al. (2004) demonstrate that many of the existing models can account only for one side of the coin: if they can explain the classical preference reversal phenomenon then they cannot accommodate empirical evidence on preference reversals with probability equivalents. 


\section{Conclusion}

The preference reversal phenomenon is an intriguing empirical regularity that continues to attract the attention of experimentalists (e.g. Bateman et al., 2007) and theorists alike (e.g. Schmidt et al., 2008). Several authors, most notably MacCrimmon and Smith (1986) and Butler and Loomes (2007), argued that preference reversals may be a reflection of imprecise certainty/probability equivalents. This paper presents a formal model that builds on such ideas.

As a starting building block, we take a model of probabilistic choice recently axiomatized by Blavatskyy (2008). This model has many intuitive features. In particular, the model rules out violations of transparent dominance. This model of binary choice is augmented by a novel definition of a probabilistic certainty/probability equivalent of a lottery. The certainty equivalent of a lottery is defined as a random variable such that the probability of the certainty equivalent being less than or equal to $x$ is simply equal to the probability that the amount $x$ is chosen over the lottery in a direct binary choice.

The probability equivalent of a lottery $A$ is defined in a similar way. It is a random variable such that the likelihood of the probability equivalent being less than or equal to $q$ is equal to the likelihood that a benchmark two-outcome lottery that yield a higher outcome with probability $q$ is chosen over the lottery $A$ in a direct binary choice. Note that these proposed definitions do not depend on a specific model of probabilistic choice that we used in this paper. They can be also combined with a random utility model (e.g. Gul and Pesendorfer, 2006), a random preference model (Loomes and Sugden, 1995) or an incremental expected utility advantage model (Fishburn, 1978).

Since an individual does not violate transparent dominance the distribution of the certainty equivalent of any lottery is restricted to have its support between the lowest and the highest possible outcome of the lottery. Furthermore, if the \$-bet and the 
P-bet yield similar expected utility, median certainty equivalents of the two bets are similar as well. All in all, this implies that the certainty equivalent of the $\$$-bet is positively skewed but the certainty equivalent of the P-bet is negatively skewed. Hence, an individual may value the \$-bet over the P-bet more frequently than vice versa.

In fact, the proposed model can also rationalize strong preference reversals when an individual chooses the P-bet over the $\$$-bet in a direct binary choice but values the \$-bet more than the highest possible outcome of the P-bet. The paper also shows that the model can account for a higher incidence of nonstandard preference reversals with probability equivalents. These occur when an individual chooses the $\$$-bet over the P-bet in a direct binary choice but states a higher probability equivalent for the P-bet.

Theoretical results of this paper clearly demonstrate that probabilistic choice models have a significant potential for explaining behavioral regularities. While the preference reversal phenomenon is a puzzling observation in the classical world of deterministic preferences, it is not really a phenomenon once we admit the possibility that individual preferences are somewhat fuzzy or imprecise. A simple model of fuzzy preferences can generate systematic preference reversals in different elicitation methods that are formally equivalent under the assumption that an individual has a unique preference relation.

This conclusion contrasts with a more pessimistic conclusion of Cubitt et al. (2004) who conjectured that "a stochastic variant of GET [generalized economic theory] is not an obviously promising line of attack" for explaining a high incidence of standard preference reversals for certainty equivalents simultaneously with a high incidence of nonstandard preference reversals for probability equivalents. It appears that Cubitt et al. (2004) considered only the possibility of heterogeneous errors across individuals but not 
across elicitation tasks. In other words, they did not envisage that there may be a model of probabilistic choice that generates the distributions of certainty equivalents and probability equivalents, which are skewed in the opposite directions.

The model presented in this paper has exactly this feature. In particular, the distribution of the certainty equivalent of the $\$$-bet is positively skewed but the distribution of the probability equivalent of the \$-bet is likely to be negatively skewed. At the same time, the opposite holds for the P-bet. Its distribution of the certainty equivalent is negatively skewed but its distribution of the probability equivalent is positively skewed. Such skewness in the opposite directions allows our model to explain overpricing of the \$-bet simultaneously with a high incidence of nonstandard preference reversals for probability equivalents.

For economists working with empirical data on binary choices between risky lotteries it long became customary to embed a deterministic decision theory into a model of probabilistic choice (e.g. Hey and Orme, 1994). Perhaps, it is now time to abandon the idea of people having deterministic certainty/probability equivalents for risky gambles. The theoretical model presented in this paper offers a unified approach for describing the decisions of an individual whose binary choices and stated valuations are both done in a probabilistic manner.

The presented model can be easily applied in empirical work where a data set contains both revealed binary choices and stated valuations (in the form of certainty or probability equivalents). Using a one-parameter functional form for function $\varphi($.$) such$ as $\varphi(x)=x^{a}$ or $\varphi(x)=\exp (a \cdot x)-1, a=$ const, a researcher obtains a parsimonious model with only one extra parameter compared to neoclassical expected utility theory. Such model may have considerable descriptive merit since it can account for the common ration effect, violations of the betweenness and the preference reversal phenomenon. 


\section{References}

Allais, Maurice (1953) “Le Comportement de l'Homme Rationnel devant le Risque:

Critique des Postulats et Axiomes de l'Ecole Americaine" Econometrica 21: 503546

Ballinger, T. Parker and Nathaniel T. Wilcox (1997) “Decisions, error and heterogeneity" Economic Journal 107: 1090-05

Bateman Ian, Brett Day, Graham Loomes and Robert Sugden (2007) “Can ranking techniques elicit robust values?" Journal of Risk and Uncertainty 34, 49-66

Blavatskyy, Pavlo (2006) "Violations of Betweenness or Random Errors?” Economics Letters 91: 34-38

Blavatskyy, Pavlo (2007) "Stochastic Expected Utility Theory” Journal or Risk and Uncertainty 34: 259-286

Blavatskyy, Pavlo (2008) "Probabilistic Choice and Stochastic Dominance" IEW Working Paper 364, available at http://www.iew.uzh.ch/wp/iewwp364.pdf Blavatskyy, Pavlo, and Wolfgang Köhler (2007) "Range Effects and Lottery Pricing" IEW working paper 323, available at http://www.iew.uzh.ch/wp/iewwp323.pdf

Butler, J. David and Graham C. Loomes (2007) "Imprecision as an Account of the Preference Reversal Phenomenon" American Economic Review 97(1): 277-297

Cubitt, Robin P., Alistair Munro, and Chris Starmer (2004) “Testing Explanations of Preference Reversal” Economic Journal 114(497): 709-26

Fishburn, Peter (1978) A probabilistic expected utility theory of risky binary choices, International Economic Review 19, 633-646

Fishburn, Peter (1988) "Nonlinear Preference and Utility Theory” Brighton: Wheatsheaf Books Ltd 
Gul, Faruk and Wolfgang Pesendorfer (2006) "Random Expected Utility" Econometrica 71(1): 121-146

Hey, John (2001) “Does repetition improve consistency?” Experimental Economics 4, 554

Hey, John and Chris Orme (1994) "Investigating Generalizations of Expected Utility Theory Using Experimental Data” Econometrica 62: 1291-1326

Lichtenstein, Sarah and Paul Slovic (1971) "Reversals of Preference between Bids and Choices in Gambling Decisions“ Journal of Experimental Psychology 89: 46-55 Loomes, Graham and Robert Sugden (1987) "Some Implications of More General Form of Regret Theory" Journal of Economic Theory 41(2): 270-287

Loomes, Graham and Robert Sugden (1995) "Incorporating a stochastic element into decision theories" European Economic Review 39, 641-648

Loomes, Graham and Robert Sugden (1998) “Testing different stochastic specifications of risky choice" Economica 65, 581-598

MacCrimmon, Kenneth and Maxwell Smith (1986) "Imprecise Equivalences: Preference Reversals in Money and Probability", University of British Columbia Working Paper 1211

Seidl, Christian (2002) “Preference Reversal” Journal of Economic Surveys 16: 621-655

Schmidt, Ulrich and John D. Hey (2004) “Are Preference Reversals Errors? An Experimental Investigation” Journal or Risk and Uncertainty 29(3): 207-218

Schmidt, Ulrich, Chris Starmer and Robert Sugden (2008) “Third-generation prospect theory" Journal or Risk and Uncertainty 36(3): 203-223

Tversky, Amos, Paul Slovic and Daniel Kahneman (1990) “The Causes of Preference Reversal” American Economic Review 80(1): 204-217 